\title{
Studying the effect of fiber size and fiber loading on the mechanical properties of hybridized kenaf/PALF-reinforced HDPE composite
}

\begin{abstract}
Hybridization, especially where only variant natural lignocelluloses are combined, is fast receiving encouraging attention because it offers a range of properties that may be difficult to obtain with a single kind of reinforcement. In this study, tensile, flexural, and impact properties of hybridized kenaf/PALF-reinforced HDPE composite were studied. Sheets from which tensile, flexural, and impact specimens were cut out were produced from the compression molding of composite pellets and subsequently conditioned in an oven for $21 \mathrm{~h}$ at $103^{\circ} \mathrm{C}$. The tensile and flexural specimens were tested according to ASTM D638 and ASTM D790 using a 5-kN INSTRON bluehill universal testing machine accordingly. While a notched Izoid impact test was conducted using a 1-J universal pendulum according to ASTM D256. All specimens were prepared at a fiber ratio of 1:1 kenaf:PALF and fiber lengths of $0.25,0.5,0.75$, and $2 \mathrm{~mm}$; fiber loadings of between $10 \%$ and $70 \%$ were utilized for the study. About $0.25 \mathrm{~mm}$ fiber showed the best tensile and flexural properties with a linear increase in properties up to $60 \%$ fiber loading while impact strength showed better property with 0.75 and $2 \mathrm{~mm}$ fiber lengths. At $0.25 \mathrm{~mm}$ fiber length, tensile strength of 32.24 $\mathrm{MPa}$, flexural strength and modulus of $34.01 \mathrm{MPa}$ and 4114.11 $\mathrm{MPa}$, respectively, were observed at $60 \%$ fiber loading. Moduli results of all the composites formulated generally obeyed the ROM. SEM was used to examine the surface of composites produced. Tensile and impact strengths result showed inverse proportionality while flexural strength of the composite generally adhered to the ROM. However, a positive effect was observed in the case of compositeôs impact strength in respect of increasing fiber length. Thus, reduction in some mechanical properties of the composite with respect to increasing fiber length is attributed more to fiber entanglement rather than attrition.
\end{abstract}

Keyword: Hybridization; Length; Loading; Modulus; Study; Tensile strength 